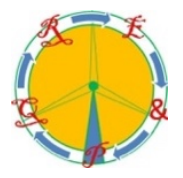

\title{
The Residual Load Duration Curve (rLDC) to model an energy system
}

\author{
W.-G. Früh \\ Institute of Mechanical, Process and Energy Engineering, School of Engineering and Physical Sciences, \\ Heriot-Watt University \\ Riccarton, Edinburgh EH14 4AS (Scotland, UK) \\ Phone/Fax number: +0049 1314514374 / 3129, e-mail: w.g.fruh@hw.a.c.uk
}

\begin{abstract}
This paper develops a framework to represent the action of different generation and balancing technologies based on the statistics of the demand and generation using the concept of the Load Duration Curve (LDC). The aim is to capture the statistics in terms of energy and power provision from a generation type given by its inherent resource, technical and economic constraints.

This paper provides a brief overview of the fundamental types of generation and balancing, and how their action modifies the load duration curve to eventually arrive at a balanced system. Based on empirical data from the UK National Grid, the statistical properties of renewable energy sources and of the energy and power capacities of energy storage technologies, different basic functional relationships between the residual Load Duration Curves (rLDC) will be derived for these technologies.
\end{abstract}

\section{Key words}

Energy system, Renewables integration, Load Duration Curve, Equivalent Load Duration Curve.

\section{Introduction}

Modelling a power system, or even a whole energy system, is often based on balancing supply and demand at a large set of time points or time slices (e.g., the TIMES model [1] or EnergyPlan [2]) which is a substantial task requiring substantial resources. At the other end, estimates are often based on balancing of bulk energy production and supply over a longer period. Such a simple energy balance can be a useful guide for contribution of a particular resource to a system and is frequently used to quantify the value of a particular project (A typical example illustrates the value of Whitelee wind farm in terms of 'Its 215 turbines generate up to 539 megawatts of electricity, enough to power just under 300,000 homes*'[3]). However, this statement fails to address the simultaneous constraints of not only balancing energy over a period but also power at every point in time. The coupling of the power and energy constraints become significant with higher contribution of renewable to energy supply.

The Load Duration curve (LDC) is a widely used statistical diagnostic of a power system. It shows for how much of a specified time (usually a year), the load exceeds a certain value [4]. Formally, it is the quantile function but in reverse (the likelihood of demand exceeding a value) or $L D C(q)=(1-\Phi(L))^{-1}$ with $\Phi$ as the cumulative distribution function of demand. It not only illustrates the overall profile but also highlights the extent and likelihood of extreme cases which cause a disproportionately high cost for the system operator.

\section{A. The residual Load curve for the UK National Grid}

The information can also be refined into the proportion of the different supply sources, where the contribution from a generation type is shown as the difference between the duration curves before and after the generation from that type has been subtracted from the demand. This is shown in Figure 1 with a typical LDC for the UK transmission grid for the year 2017 [5]. It shows that a total system demand above $50 \mathrm{GW}$ is observed only for a very small (but not insignificant) fraction of the year, while demand in excess of around $30 \mathrm{GW}$ for around half of the year, and demand between around $21 \mathrm{GW}$ and $30 \mathrm{GW}$ for the remainder of the year. Breaking this down, from top to bottom, transmission-level Photovoltaic power moves the LDC to the first residual LDC ( $r L D C$ ) as the boundary between the yellow and green areas in Fig. 1. As the load is not reduced at small $q$, PV did not contribute to reducing the highest of the peak demand. At the other end, near $q=1$, the $r L D C$ drops off more than the $L D C$. This suggests that the feed-in of PV power occurred disproportionally more at times of low demand. 


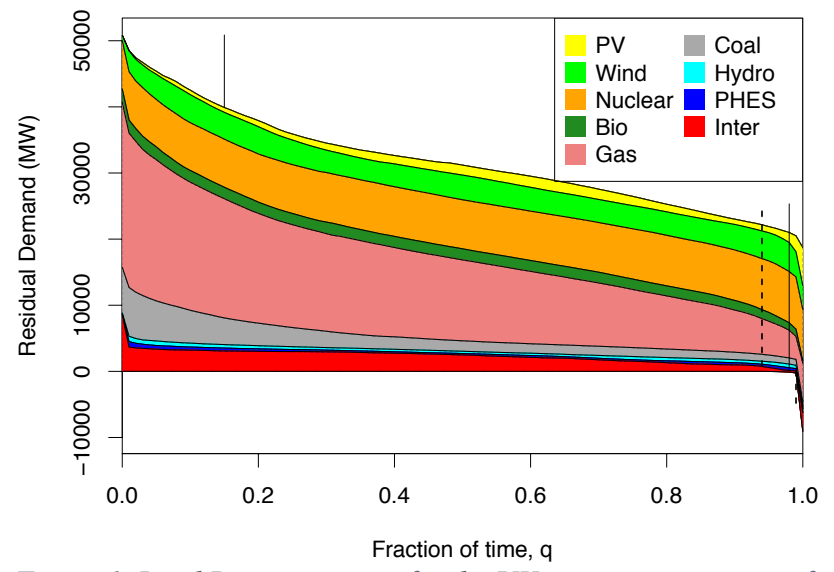

Figure 1. Load Duration curve for the UK transmission system for 2017 (Data source: [2]).

At next order, wind power (green) reduced demand more evenly across the time but still did not reduce the maximum peak demand. Nuclear (orange) and biomass (dark green) as base-load provisions shift the entire curve downwards. Gas-fired plant provided not only the bulk of generation but also met peak demand. One of the consequences of significant wind penetration and the high solar penetration during load demand is the occurrence of a net negative load.

While Fig. 1 is based on published demand and generation data, it is also possible to define theoretical or projected $L D C$ for the purpose of modelling energy systems, sometimes referred to as the concept of the Equivalent Load Duration Curve [6]. These can be based on polynomials or some statistical distributions [7,8]. As Fig.1 suggests, base-load generation shifts the LDC down without changing its shape significantly, while load-following plant and balancing mechanisms are able to flatten the residual load duration curve by meeting more of the peak demand and reducing output at low demand, or even providing a power sink at times of negative residual demand (ie, surplus renewable generation).

\section{Modelling approach}

The aim of this paper is to develop statistical models of the effect of the fundamental types of generation and balancing. Their formulation is here guided by the UK transmission grid date for 2017, observed at 5-minute intervals. This is used to propose relationships between residual demand to be addressed by a generation type and their standard response. This requires a hierarchy following the Merit Order curve, with variable Renewables leading the supply, followed by base-load generation, then load-following generation and finally fast response and balancing. Of particular interest for systems with a high renewable penetration are balancing mechanisms which allow timeshifting of demand and supply.

The formal approach is to take the demand as given (ie, the UK demand), and then to create the observed residual LDC following the current merit order. The observations are then used to propose a function, $f_{1}$, to link the first generation type, $P_{1}$, to the $L D C$,

$$
r L D C_{1}=f_{1}\left(L D C, P_{1}\right)
$$

The same procedure continues through the merit order of generation types,

$$
r L D C_{i}=f_{i}\left(r L D C_{i-1}, P_{i}\right)
$$

\section{A. Variable Renewables}

Since these types of generation are not load following (apart from the option to curtail output), they are not causally linked to demand. As a result, the effect of their production to reducing demand is based on the joint probability of a given demand being reduced by a certain output. In the simplest case, generation and demand are sufficiently independent, in which case the joint probability reduces to the product of the distribution functions. Therefore, the function $f_{\mathrm{i}}$ in equations (1) and (2) involves the construction of the distribution function from $L D C$ or $r L D C_{i-1}$ and the distribution function of the renewable generation to calculate the joint distribution, and then construct $r L D C_{i}$ from that.

A first attempt by [9] assumed that wind contribution was uniformly distributed. While this might be a reasonably good approximation for the bulk of the $r L D C$, it is bound to fail at the ends of the distribution since wind power is much better described by a beta-distribution [10]. The shape of the distribution at its extremes (no output or full output) will emerge to be an important aspect.

\section{B. Dispatchable generation}

Since these types of generation are load following but with an elasticity given by their technological and financial constraints, it is postulated that their output is a function of the residual demand to be met after the generation earlier in the merit order. To first order, this can be approximated by a piece-wise linear response to the residual load, with output at installed capacity for highest residual load, and minimum or zero output at lowest or negative residual load. A result of this approximation and assumption, $f_{\mathrm{i}}$ in equation (2) is a simple piece-wise linear function.

\section{B. Balancing technologies}

At a 'power' level, these technologies can either provide power during high residual demand, or absorb power during low (or even negative) residual demand. The three main representatives are

1. electricity import or export through interconnectors,

2. electricity storage, and

3. demand-side management.

Interconnectors can be modelled as a zero-loss generation or load up to their capacity, $\pm G_{I}$, where the main driver for operation would be market prices in the local system and the connected system. To first order, it can be modelled as a linear function of the $r L D C$, similar to load-following generation. 
Electricity storage is not only a 'lossy' balancing mechanism but a technology which is limited in both, power rating and energy capacity. As a result, the installed storage can only absorb a limited amount and keep that only for a limited time. The amount for one cycle of power absorption and provision defines the area above the $r L D C_{i-1}$ (at the low-demand end). The fraction of that area after taking into account the round-trip efficiency of the technology, can then be used to remove that area under the $r L D C_{i-1}$ at the high-demand end. Unless there is substantial seasonal energy storage, this 'area-shifting' is limited to areas associated with periods close in time. The $L D C$ itself does not give any indication as to when a specific load occurs, and the development of an appropriate shifting function needs to refer to the time series of residual load. The two sets of vertical lines indicate seasonal separators. The solid vertical line at $q \sim 0.18$ indicates that all loads above (and to the left) that point occurred during the winter months from October to March, while those to the right of $q=0.98$ occurred during the summer months. The dashed lines at $q=0.95$ and 0.99 , respectively, show the seasonal separation for the residual load at the point where pumped hydro energy storage is invoked. One message in comparing the two sets of seasonal separators is that the integration of Renewables exacerbates the seasonal mismatch between generation and demand.

Demand-side management is a loss-less load shifting but only operates across very short time periods, from withinhour shifting to a few hours at most.

\section{Residual Load Duration Curve models}

In this section, the models used to represent the different types of generation are outlined. The rLDC derived from these models are then compared against the observations.

\section{A. Solar Power}

The PV output has a Pearson's correlation coefficient with the demand of $r=-0.035$, which suggests such a weak correlation that they can be treated as independent (for the scope of this paper). As a result, the distribution $\varphi_{1}$ of the residual load, $r$, can be estimated as

$$
\varphi_{1}(r) d r=\int_{0}^{G_{1}} \varphi_{0}\left(r+r^{\prime}\right) p_{1}\left(r^{\prime}\right) d r^{\prime}
$$

with $\left(\min D-G_{1}\right) \leq r \leq \max D$, where $D$ is the load, $G_{1}$ the PV installed capacity, $\varphi_{0}$ the distribution of the load, and $p_{1}$ the distribution of solar power output.

\section{B. Wind Energy}

The wind power production also has only a weak correlation with demand $(r=0.12)$, and can be approximated as a beta distribution with $\beta_{1}=1.47$ and $\beta_{2}=2.50$. Applying eq.(3) but now using the standard beta-distribution instead of the empirical distribution, reduces $r L D C_{1}$ to $r L D C_{2}$ as shown in Fig. 3.
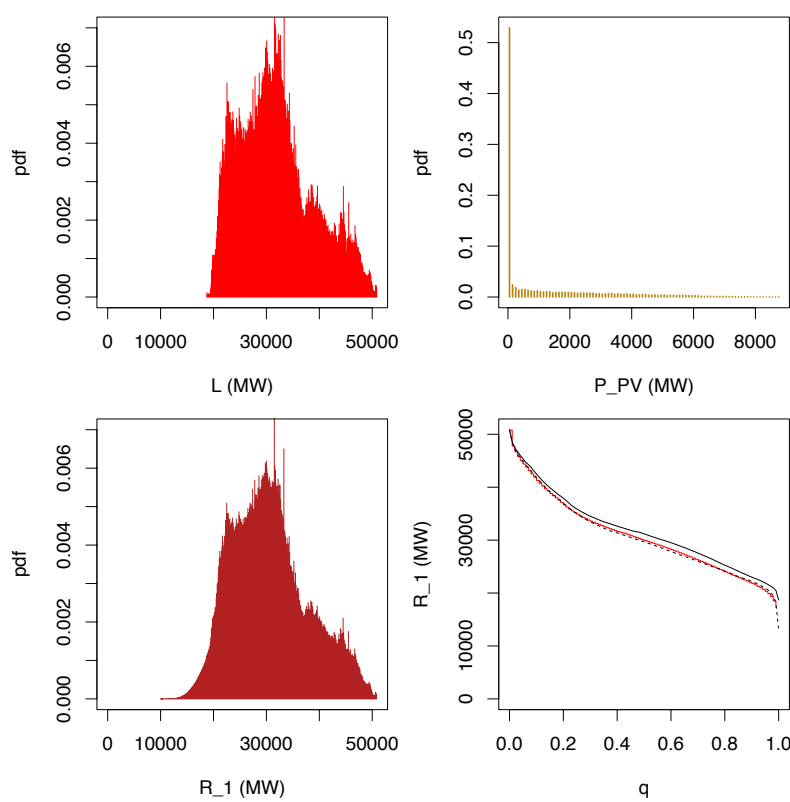

Figure 2. (a) Load distribution, (b) PV distribution, (c) distribution as calculated from eq. (3), and (d) converted to rLDC (in red), together with the LDC (black solid line) and the observed rLDC (black dashed line).
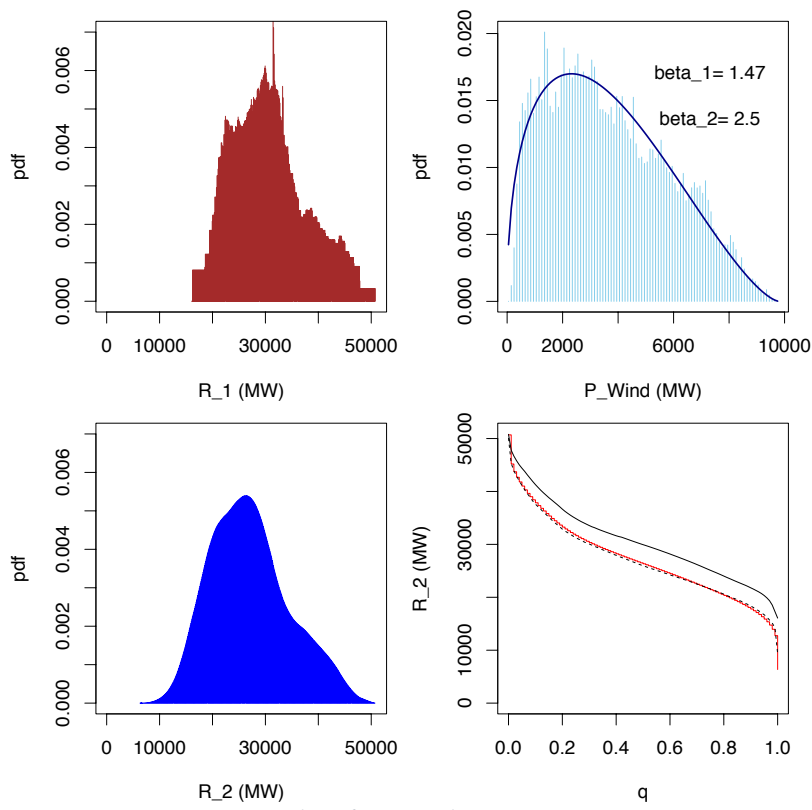

Figure 3. As Figure 2, but for Wind power.

\section{Dispatchable Generation}

For the scope of this paper, the behaviour of the dispatchable generation observed in the UK system was used to derive very simple linear relationships. Nuclear power was found to vary between $87 \%$ and $100 \%$ of its installed capacity but that this variability had no correlation with the load. Therefore, nuclear power was treated exactly the same as the solar power contribution, using eq.(3) and the observed generation distribution.

Since around 2016, the merit order curve in the UK has increasingly demoted coal to beyond biomass and CCGT [11]. These three showed a linear response but with two distinct slopes, and some with constant sections as illustrated in Fig.4. 


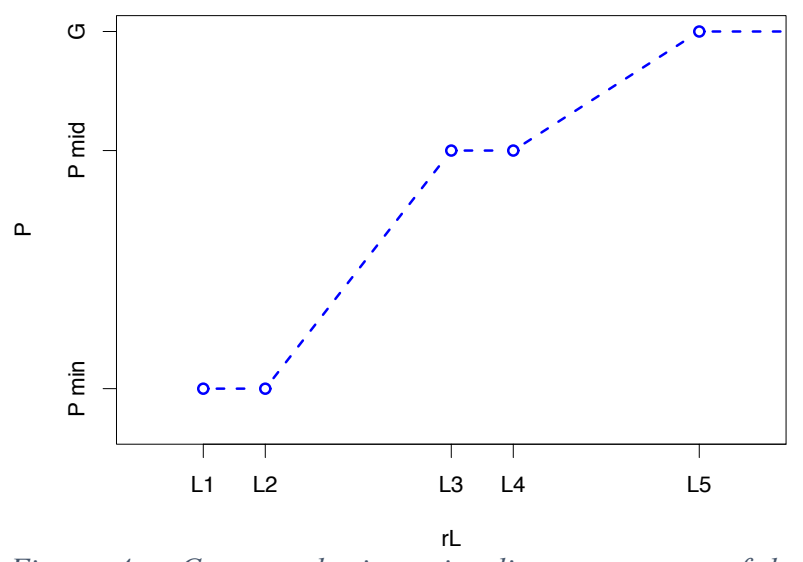

Figure 4. Conceptual piece-wise linear response of loadfollowing generation: power response, $\mathrm{P}$, vs residual load, $\mathrm{rL}$.

The response of generation $i, P_{i}(q)$ to $r L D C_{i-1}(q)$ was calculated using Fig. 4 with the installed capacity, $G$, and the parameters $L_{1} \ldots 5$ and $P_{\text {min }}, P_{\text {mid }}$, as listed in Table I (shown at the end of the paper) in their order of merit used for the modelling. The next-stage $r L D C$ was then calculated as

$$
r L D C_{i}(q)=r L D C_{i-1}(q)-P_{i}(q) .
$$

The result of successively applying these linear models is shown in Fig.5.

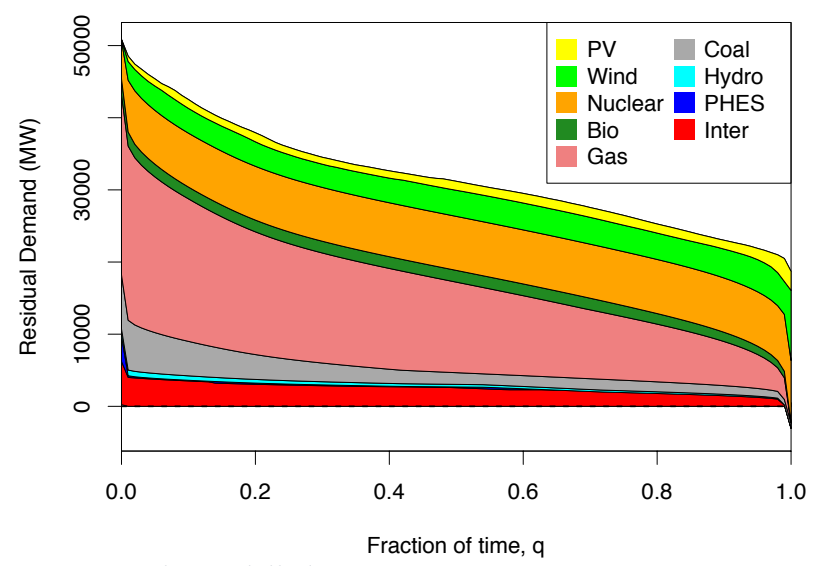

Figure 5. The modelled rLDC scenario up to interconnectors and hydropower contribution for the 2017 portfolio of generation.

\section{Energy Storage}

The fundamental function of energy storage is to absorb available power during times of low or negative residual load and use that to reduce peak residual load. In terms of the $r L D C$ curve, the 'right end' would be lifted up while the 'left end' of the curve would be lowered. This section proposes a way to quantify this effect based on the installed power generating capacity, $G_{S}$, and energy storage, $C_{S}$.

The initial step is to quantify the amount of energy which the available storage can provide over a year (or the period analysed). For this, the duration of discharging the available stored energy, $C_{S}$, at capacity is calculated as $C_{S} / G_{S}$. If the discharge period is less than 12 hours, it is assumed that one full cycle can be completed per day, or
365.25 cycles per year, and the total energy potentially supplied is $E_{S, \max }=365.25$ Cs. For installations with a longer discharge period, the number of cycles is correspondingly reduced,

$E_{S, \max }=N_{S} C_{S}$ with $N_{S}=\left\{\begin{array}{l}365.25 \text { if } C_{S} / G_{S}<12 \mathrm{~h} \\ 4383 \mathrm{~h} G_{S} / C_{S} \text { otherwise }\end{array}\right.$.

This energy is equal to an area of $G_{S} 8766 \mathrm{~h} \Delta q$. If the storage were used to its capacity, the effect of discharging at peak times across the year would therefore reduce the section of the $r L D C$ from $q=0$ to $q=\Delta q_{S}$ by $G_{S}$ with

$$
\Delta q_{S}=\frac{E_{S, \max }}{G_{S} 8766 \mathrm{~h}}
$$

To supply that energy, the energy absorbed by the recharging or pumping has to take into account the roundtrip efficiency, $\eta_{R T}$, of the storage, resulting in an additional energy consumption of $D_{S, \max }=E_{S, \max } / \eta_{R T}$ at times of low residual load, or an increase of the $r L D C$ by $G_{S}$ from $q=\left(1-\Delta q_{S} / \eta_{R T}\right)$ to $q=1$.

Applying this shift downwards of the $r L D C$ at high load and the matching shift upwards at low load has to be capped such that the shift upward at one end does not lead to a new demand higher than the resulting shift downward at the other end - ie, applying this shifting only to achieve peak shaving and trough filling without turning the original trough into a new peak and the old peak into a trough.

A final refinement of this procedure for the purpose of this paper is to recognise that peak loads are usually associated with winter conditions and low loads or high renewable generation by PV is associated with summer conditions. This means that the peak shaving of the most extreme winter peaks can only affect the low-load conditions also observed in the winter. Referring back to Fig.1, the demand above $40 \mathrm{GW}$, to the left of the vertical line at $q \sim 0.18$ is only observed in winter, and the demand below $25 \mathrm{GW}$ to the right of the vertical line at $q \sim 0.98$ is only observed in the summer. This means that the shifting has to be split into seasons such that half of the energy calculated in eq.(5) is used to balance the winter peaks from $q=0$ against the load to the left of the 'summer' line at $q \sim 0.98$, and the other half of the energy is used to balance the load immediately to the right of the 'winter' line at $q \sim 0.18$ against the far end of the $r L D C$.

After applying the shift up and down to the appropriate sections of the $r L D C$, the next-stage residual load curve is obtained by sorting the result in descending order.

Since the currently installed Pumped-Hydro (PHES) capacity in the UK system is too small $(2.8 \mathrm{GW}$ and $26.7 \mathrm{GWh}$ ) to show the effect of applying this shifting of the loads, this will be demonstrated by constructing two energy scenarios in the following section. 


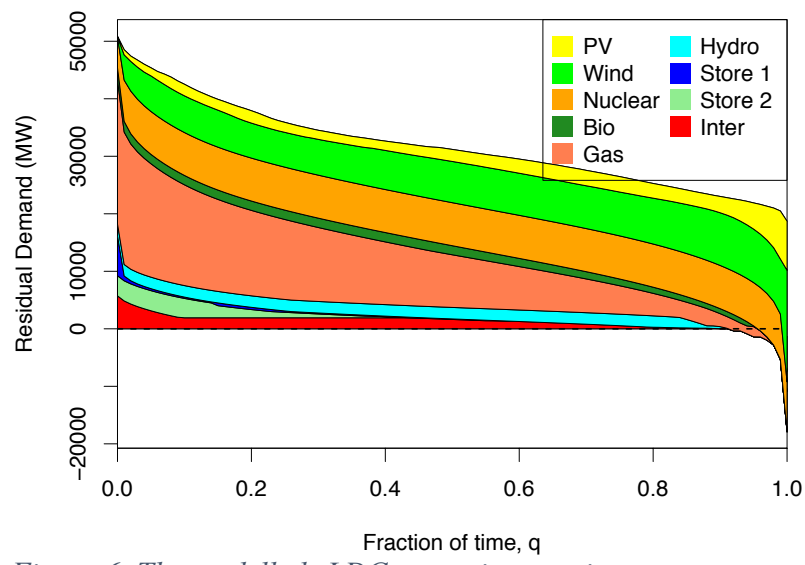

Figure 6. The modelled rLDC scenario up to interconnectors and hydropower contribution for doubled PV and Wind and zero coal.

\section{Energy scenarios}

The $r L D C$ model was applied to two energy scenarios where the demand was kept at the 2017 level but the Renewable contribution was increased while the fossil capacity was decreased. To ensure reliable supply at all times, the energy storage and interconnector capacity were adjusted. To illustrate the effect of the storage action, two types of storage were applied, first a high-power low-energy type (referred to as Store 1) and secondly a low-power high energy type (Store 2).

\section{A. Zero Coal}

In the first scenario, all coal generation was eliminated but PV, Wind, and Hydro capacity were doubled compared to 2017. The high-power low-energy plant was based on an installed capacity of $7 \mathrm{GW}$ (compared to the existing PHES $2.8 \mathrm{GW}$ ) and $10 \mathrm{GWh}$, or an energy/power ratio of 1.4 hours. The low-power high-energy type of storage was set at $3.5 \mathrm{GW}$ and $100 \mathrm{GWh}$ (28.57 hours). To balance the system, the interconnector also had to be increased from $4 \mathrm{GW}$ to $6 \mathrm{GW}$ (see Table I).

The results shown in Fig.6 demonstrate that the high-power low-energy storage type 1 does effectively reduce the highest of the peak demand but has little effect for the rest of the year. In contrast, the low-power high-energy Storage type 2 cannot fully shave all the peaks but has a visible effect of levelling the residual load for a substantial part of the time. Since the net energy produced by the renewable installation and scheduled generation in the current order of analysis is not sufficient to meet the total consumption, the 'Store 2' balances the system towards a flat but positive residual load.

In the present model formulation, this residual load is then taken by the interconnector. However, a revised model could include a further iteration step, revisiting the scheduled generation from biomass and gas, in addition to utilising the interconnector. This step could allocate the residual load between unused generation capacity and import based on pricing signals estimated from the margin between demand and capacity.

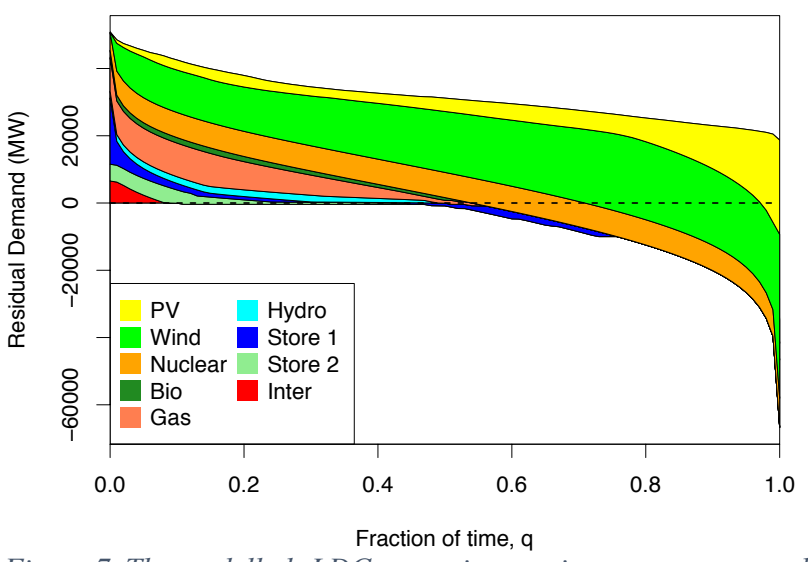

Figure 7. The modelled rLDC scenario up to interconnectors and hydropower contribution for reduced Gas capacity.

\section{B. Reduced Gas}

In the second scenario, shown in Fig.7, the installed capacity of the gas-powered generators is reduced from $25 \mathrm{GW}$ to $10 \mathrm{GW}$ (see Table I). A system balance was achieved by increasing the storage type 1 to $20 \mathrm{GW}$ and $28 \mathrm{GWh}$ (maintaining the 1.4 hours ratio). Storage type 2 was doubled to $7 \mathrm{GW}$ while its energy capacity did not need to be increased, reducing the discharge period to 14.29 hours. To balance the system, the interconnector had to be increased from $6 \mathrm{GW}$ to $9 \mathrm{GW}$.

\section{Discussion and Conclusion}

Overall, the $r L D C$ models are able to reproduce the observed $r L D C$ very well, in particular the distribution of the high-load events, which are usually the most critical times and associated with the highest costs.

The statistical approach of the joint probabilities has been successful not only in reproducing the overall shape of the $r L D C$, but especially at the ends of the curve. The two renewable resources, solar with the distribution skewed to many hours of no output and wind with low likelihood of zero or full output, have reproduced the key features that the maximum peak demand is unlikely to be reduced by those resources but that it reduces minimum demand significantly. Likewise, assuming nuclear generation to be distributed between two finite values, has successfully shifted the $r L D C$ downwards consistent with observations.

\section{A. Further work}

For the purpose of developing the framework observed distributions and best-fit standard distributions were used. For modelling of future energy scenarios, it is important to define appropriate general distribution functions. Further work therefore is to define a suitable distribution function for solar power and other generation types which are not load-following. The assumption of independence between demand and generation was found to be acceptable for a Pearson's correlation coefficient of up to 0.12 . Further work is needed to establish the range of validity of this assumption and to develop a method to calculate the joint probability for correlated distributions. 
The piece-wise linear model of the dispatchable generation and interconnectors has reproduced the overall effect well enough to be a promising approach. It is an attractive approach not only because of the simplicity of the mathematics but because it is also easy to associate distinct features of the functions with technological constraints (such as ramp rate and minimum operating point) as well as financial factors (by the change in the slope of the response, for example). For the scope of this work, however, the values determining the different responses were set purely by fitting the observed response to the postulated function. In future scenarios, it is likely that the response of a generation type will have to adjust to the new conditions. For example, nuclear generation might need to become more load following if it becomes the main dispatchable generation [12,13]. Hence, further work is needed to establish a better understanding of how different operating modes, technical constraints and economic signals affect the parameter settings for the dispatchable generation modelling.

Finally, the energy storage modelling is a first proposal which will need to be tested, for example by applying the method to a variety of systems or comparing its performance against the more established energy systems models.

\section{References}

[1] R. Loulou, G. Goldstein, K. Noble, "Documentation for the MARKAL family of models, ETSAP, 2004., see also https://iea-etsap.org/index.php/etsap-tools/modelgenerators/times, accessed 18 Jan 2019.

[2] H. Lund, J.Z. Thellufsen, "EnergyPLAN: Advanced Energy Systems Analysis Computer Model", Documentation V.14, 2018, see also https://www.energyplan.eu/, accessed 18 Jan 2019.

[3] Scottish Power, Whitelee Windfarm website, https://www.scottishpower.co.uk/whitelee/, accessed 18 Jan 2019.
[4] A. Poulin, M. Dostie, M. Fournier, S. Sansregret, "Load duration curve: A tool for technico-economic analysis of energy solutions", Energy and Buildings, Vol. 40, pp. 29 35.

[5] ELEXON, "Historic generation by fuel type", www.elexonportal.co.uk/historicgenerationbyfueltype, accessed 19 Nov 2017.

[6] A.S. Malik, B.J. Cory, "An application of frequency and duration approach in generation planning", IEEE Transactions on Power Systems, Vol. 12, pp. 1076- 1084.

[7] M. Ould Mohamed Mahmoud, M. Jaïdane-Saïdane, J. Souissi, N. Hizaoui, "Modeling of the load duration curve using the asymmetric generalized Gaussian distribution: Case of the Tunisian power system", in IEEE Power and Energy Society 2008 General Meeting: Conversion and Delivery of Electrical Energy in the 21st Century, PES.

[8] M. Kato, Y. Zhou, C. Kang, R. Yokoyama, "Novel approach of modeling load duration curve for generation expansion planning based on Hill's function", in IEEJ Transactions on Electrical and Electronic Engineering, 2011, Vol. 6, pp. 304-310.

[9] A. Wiskich, "Implementing a load duration curve of electricity demand in a general equilibrium model", in Energy Economics, 2014, Vol. 45, pp.373-380.

[10] W.-G. Früh, "From local wind energy resource to national wind power production", in AIMS Energy, 2015, Vol. 3, pp.101-120.

[11] Ofgem, "Wholesale Energy Markets in 2016", 2016, https://www.ofgem.gov.uk/system/files/docs/2016/08/wh olesale energy_markets in 2016.pdf , accessed 18 Jan 2019.

[12] Nuclear Energy Agency, "Technical and economic aspects of load following with Nuclear power plants, OECD, 2011, https://www.oecd-nea.org/ndd/reports/2011/loadfollowing-npp.pdf, accessed 18 Jan 2019.

[13] S.R. Greene, "Enhancing Electric Grid, Critical Infrastructure, and Societal Resilience with Resilient Nuclear Power Plants (rNPPs)", in Nuclear Technology, 2018, Vol. 204, pp.1-18.

Table I. - Dispatchable Generation parameters as estimated from the UK National Grid data in 'merit order'. The parameters are scaled against the installed capacity. If $L_{1}$ is absent, no minimum plateau was observed. If $L_{4}$ is absent, no intermediate plateau was observed. If $L_{5}$ is not given, no intermediate plateau was observed and $L_{5}=\max (r L)$. If $P_{\text {mid }}, L_{4}$ and $L_{4}$ are not given, the response is simply linear with a single slope. A negative $L_{1}$ indicates that generation continues even at times of generation surplus, and $L_{1}=-\infty$ for the interconnector indicates that it exports at its capacity for all excess generation above its export capacity.

\begin{tabular}{|c|c|c|c|c|c|c|c|c|}
\hline $\begin{array}{l}\text { Generation } \\
\text { type }\end{array}$ & $\begin{array}{c}\text { Capacity } \\
G \\
(\mathrm{GW})\end{array}$ & $\begin{array}{l}P_{\min } \\
(G)\end{array}$ & $\begin{array}{c}P_{\text {mid }} \\
(G)\end{array}$ & $\begin{array}{l}L_{1} \\
(G)\end{array}$ & $\begin{array}{l}L_{2} \\
(G)\end{array}$ & $\begin{array}{l}L_{3} \\
(G)\end{array}$ & $\begin{array}{l}L_{4} \\
(G)\end{array}$ & $\begin{array}{l}L_{5} \\
(G)\end{array}$ \\
\hline Nuclear & 8.66 & 0.87 & & & & & & \\
\hline Biomass & 2.17 & 0.4 & 0.75 & 0.5 & 2 & 7 & 14 & 20 \\
\hline Gas (CCGT) & 25 & 0.12 & 0.55 & 0.03 & 0.2 & 0.75 & & 1.5 \\
\hline Coal & 7.5 & 0.1 & 0.2 & -1 & 0.2 & 0.5 & 0.6 & 1.7 \\
\hline Hydro & 1 & 0 & 0.35 & & 0 & 3 & & 6 \\
\hline Interconnector & 4 & -1 & & $-\infty$ & -1 & & & 1 \\
\hline \multicolumn{9}{|c|}{ Zero Coal scenario } \\
\hline Hydro & 2 & 0 & 0.35 & & 0 & 0.5 & & 1 \\
\hline Interconnector & 6 & -1 & & $-\infty$ & -1 & & & 1 \\
\hline \multicolumn{9}{|c|}{ Reduced Gas scenario } \\
\hline Gas (CCGT) & 10 & 0.12 & 0.55 & 0.03 & 0.2 & 0.75 & & 1.5 \\
\hline Hydro & 2 & 0 & 0.35 & & 0 & 0.5 & & 1 \\
\hline Interconnector & 9 & -1 & & $-\infty$ & -1 & & & 1 \\
\hline
\end{tabular}

